\title{
Algunas claves para entender la actual configuración jurídica de los planes de igualdad: alcance y contenido
}

\author{
Some keys to understand the current legal configuration \\ of equality plans: scope and content
}

\author{
Carlos L. Alfonso Mellado* \\ Catedrático de Derecho del Trabajo y Seguridad Social \\ Universidad de Valencia
}

ORCID: 0000-0001-6096-0212

Recibido: 09/03/2020

Aceptado: 30/03/2020

doi: https://doi.org/10.20318/femeris.2020.5383

\begin{abstract}
Resumen. El presente trabajo analiza el alcance, significado y contenido de los planes de igualdad. Se analiza la igualdad como valor esencial en un Estado Social y la evolución en la protección jurídica de la igualdad entre mujeres y hombres en el ámbito del trabajo. Posteriormente se analiza el significado de los planes de igualdad como elemento integral para la consecución de la igualdad efectiva entre mujeres y hombres en el ámbito laboral. Seguidamente se estudian algunas características esenciales de los planes de igualdad, especialmente tras los últimos cambios normativos como la expansión de su ámbito de aplicación a más empresas, la importancia del diagnóstico como elemento esencial para delimitar las medidas a incorporar en el plan de igualdad, el carácter negociado del plan y su dinamismo que lleva a su temporalidad y a la necesidad de seguimiento a través de indicadores y, en su caso, del correspondiente replanteamiento de las medidas integradas en el plan en atención a la evolución de la situación de la igualdad efectiva entre mujeres y hombres en la empresa.

Palabras clave: igualdad de género, mujer, discriminación laboral, discriminación por razón de género, legislación antidiscriminatoria.
\end{abstract}

Abstract. This paper analyses the scope, meaning and content of equality plans. It analyses equality as an essential value in a social state and the developments in the legal protection of equality between women and men in the field of work. The meaning of equality plans is then analyzed as an integral element for achieving effective equality between women and men in the workplace. Some key features of equality plans are then explored, especially after the latest regulatory changes such as the expansion of their scope to more companies, the importance of diagnosis as an essential element in delimiting the measures to be incorporated into the equality plan, the negotiated nature of the plan and its dynamism that leads to its temporality and the need to follow-up through indicators and, where appropriate, the corresponding rethinking of the measures integrated into the plan taking into account the evolving situation of effective equality between women and men in the company.

Keywords: gender equality, women, labor discrimination, gender discrimination, nondiscriminatory legislation.

*alfonsoc@uv.es 


\section{Introducción}

Para un hombre valenciano es, sin duda, un reto reflexionar sobre los planes de igualdad, especialmente si el encargo procede de la Asociación Española de Derecho del Trabajo y Seguridad Social (AEDTSS), y ello por múltiples razones.

En primer lugar porque en el marco de la AEDTSS existen numerosas mujeres que han trabajado y muy bien los temas relativos a la igualdad entre mujeres y hombres en el ámbito laboral, sin duda mucho mejor de lo que lo podría hacer yo y a algunas haré referencia posteriormente; también otros hombres miembros de la AEDTSS lo han hecho con trabajos que son referencia imprescindible como el profesor Cabeza Pereiro o el magistrado Lousada Arochena.

Pero, en segundo lugar y muy especialmente, por mi condición de valenciano que me hace compartir ámbito con personas que son referentes absolutos en la materia como, en el ámbito laboral, las profesoras Ballester Pastor y Fabregat Monfort, especialmente esta última en materia de planes de igualdad con obras que son referencia imprescindible como también sobre medidas de acción positiva en el ámbito laboral; o, en el ámbito del fundamento constitucional de la igualdad, con profesoras como Sevilla Merino o Ventura Franch; o, incluso en terrenos distintos del jurídico, desde el feminismo filosófico - sin entrar en el debate acerca de si debe identificarse así o como filosofía feminista - como la profesora Amorós Puente, que ha evidenciado en numerosas reflexiones las profundas raíces que sustentan la sociedad patriarcal y que hacen que se perpetúe, incluso estableciendo sujetos colectivos que, de algún modo, marginan a la mujer y reproducen esquemas sociales de desigualdad.

Y eso por citar solo algunos ejemplos.

Puede entenderse, pues, el reto que supone hablar de igualdad, siendo un hombre valenciano, cuando además las mujeres que reflexionan sobre la materia no sólo lo hacen muy bien, sino que también viven diariamente las situaciones de desigualdad, percibiéndolas incluso en matices o situaciones que se nos escapan a los hombres, precisamente por los complejos engranajes de la sociedad patriarcal que no siempre aparecen con claridad para quien no padece directamente sus consecuencias.

Pese a lo anterior la materia no me es ajena, la he tratado en anteriores ocasiones, especialmente en relación con dos aspectos: la igualdad en el empleo público y la brecha salarial.

Es más, creo que la materia debe ser de tratamiento imprescindible, también por los hombres; la igualdad no es cosa de mujeres, sino algo que nos afecta a todos; no me puedo sentir libre si la persona que está a mi lado no tiene garantizada la igualdad que es elemento esencial de la libertad.

En la construcción de una sociedad más democrática, más sostenible, más solidaria, en definitiva más justa, el papel de las mujeres es esencial y por ello garantizar su igualdad en todos los ámbitos es una pieza fundamental, posiblemente la base más importante para ese avance hacia sociedades mejores. Sin duda a ello ha de contribuir primordialmente la lucha del feminismo y de muchas mujeres; los hombres, sin protagonismo ni proteccio- 
nismo patriarcal, lo que hemos de hacer es apoyar en cuanto podamos esa lucha y a ello pretenden contribuir estas modestas reflexiones en un ámbito tan concreto como el de los planes de igualdad pero, a la vez, tan esencial en la empresa como se verá.

\section{Cuestiones previas sobre la igualdad entre mujeres y hombres}

Tras las anteriores reflexiones, más sociales que jurídicas aunque imprescindibles para situar correctamente el análisis jurídico, creo necesaria también una reflexión general, aunque breve, sobre el valor de la igualdad que permita entender el significado de los planes de igualdad y su encaje en el ordenamiento laboral. Esa reflexión pasa por dos elementos: situar el valor igualdad como elemento esencial del Estado Social y analizar la evolución de la protección jurídico-laboral de la igualdad entre mujeres y hombres, que ha llevado a la necesidad de los planes de igualdad e incluso a las reformas que han precisado su régimen jurídico.

\subsection{La igualdad y su importancia en el Estado Social}

Cualquier análisis en la materia debe partir del art. 1.1. de la Constitución española (CE) que proclama que nuestra sociedad se constituye en un Estado social y democrático de Derecho, que propugna como valores superiores de su ordenamiento jurídico la libertad, la justicia, la igualdad y el pluralismo político.

La igualdad se constituye así, desde el principio, en un valor esencial del Estado Social, pero no cualquier tipo de igualdad, sino la igualdad real y efectiva como evidencia el mandato dirigido en tal sentido a los poderes públicos (art. 9.2 CE).

Por ello, la igualdad a la que se refiere el art.14 CE es más amplia que la interdicción de la discriminación que menciona posteriormente el precepto, especialmente si se tiene en cuenta la perpetuación en el tiempo de desigualdades sociales y las profundas raíces de la sociedad patriarcal que no se combaten solamente impidiendo la discriminación hacia el futuro o las actuaciones de presente, sino que exigen actuaciones integrales, promocionales y/o equilibradoras que garanticen la igualdad de oportunidades real y efectiva entre todas las personas que componen la sociedad.

Como se ha podido exponer, en el Estado Social, el valor de la igualdad se convierte en un valor superior del ordenamiento jurídico y en un principio informador de todo él, que requiere no sólo la prohibición de la discriminación directa o indirecta, sino también la existencia de unas medidas promocionales de la igualdad, que pueden consistir en medidas de acción positiva, medidas tendentes a garantizar la igualdad de oportunidades y medidas en orden a la participación equilibrada (Lousada, 2011, pp. 25-27), y posiblemente en un conjunto de todas ellas.

Es preciso, pues, una actuación integral, seria, promocional y que compense las desigualdades históricas porque solo así se conseguirá el objetivo real del Estado Social que 
es la igualdad real, y para las mujeres la libertad entendida como ausencia de dominación. De no actuarse así, el Estado Social habrá fracasado y solo garantizara una igualdad formal, pero no real, y una libertad entendida meramente como ausencia de interferencia (Esquembre, 2018, p. 109), pero que no llegará a cuestionar los fundamentos reales de los esquemas patriarcales.

Como se ha expuesto igualmente (Casas, 2019, especialmente pp. 14-18), eso supone, sin duda una perspectiva de género, que supere la mera de igualdad por razón de sexo y que entienda que no es un hecho biológico el que esencialmente discrimina la mujer, sino las construcciones culturales, ideológicas en suma, que sustentan la sociedad patriarcal

La actuación de los poderes públicos promoviendo la igualdad real es, pues, no solo una posibilidad sino realmente una obligación constitucional, solventando así la situación de desventaja en la que en muchos casos se encuentra la mujer como consecuencia de tradiciones y hábitos profundamente arraigados en la sociedad y que, por tanto, son muy complejos de eliminar, como afirma la sentencia del Tribunal Constitucional (STC) 128/1987, de 16 de julio.

La actuación integral que garantice la igualdad real entre mujeres y hombres es esencial, pues, en la propia configuración del Estado Social y conduce a una conclusión muy clara; no puede considerarse una sociedad libre e igualitaria cuando la mayoría de las personas que la componen (las mujeres) no goza de igualdad real de oportunidades y, por tanto, no está en las mismas condiciones para usar su libertad. Nadie puede sentirse libre e igual en una sociedad en la que la mayoría de quienes la componen no lo son en toda su plenitud y al respecto los retos son aún muy grandes (un buen análisis interdisciplinar sobre estos retos en Selma (dir.), 2019).

En esa dimensión igualitaria de la sociedad es evidente que pese a los años transcurridos desde la aprobación de la CE, la igualdad real no se había conseguido y a eso respondió la Ley Orgánica 3/2007, de 22 de marzo, para la igualdad efectiva de mujeres y hombres (LOIEMH), como norma integral que pretendía dar respuesta a la insuficiencia de las medidas adoptadas hasta ese momento en la materia, sin duda provocada también esa insuficiencia por las reticencias jurídicas hacia la perspectiva de género y hacia las medidas de acción positiva (Casas, 2019, pp. 35-44).

En ese sentido se ha llegado a calificar a la LOIEMH como una auténtica norma de desarrollo constitucional que venía a intentar solventar el reiterado incumplimiento de la obligación constitucional de garantizar la igualdad real entre mujeres y hombres (García y Ventura, 2018, p. 53) y a resaltar su papel como norma que reforzaba claramente la posibilidad de las medidas de acción positiva en el ámbito laboral para completar la insuficiente tutela contra las discriminaciones (Fabregat, 2009, pp. 9 a 27), favoreciendo igualmente la perspectiva de género en materia de igualdad.

Lógicamente, la LOIEMH prestaba especial atención al ámbito laboral por dos razones muy claras: la importancia que en la vida de cualquier persona tiene el trabajo como medio de integración social y como parte muy importante de su tiempo vital; y como segunda razón, por la evidencia de que, pese a toda la regulación laboral al respecto y pese a que ciertamente se han conseguido avances en la materia, el trabajo sigue siendo un 
ámbito en el que la igualdad real no está garantizada. lo que me lleva al segundo plano de consideraciones previas, esto es a la evolución de la regulación jurídica laboral que ha llevado a la institución de los planes de igualdad.

1.2. El progresivo avance de la protección de la igualdad entre mujeres y hombres en el ámbito laboral

El camino hacia la igualdad entre mujeres y hombres en el ámbito laboral puede decirse que se inició normativamente con el retorno del Estado español al sistema democrático; en la dictadura surgida con posterioridad al alzamiento franquista la mujer ocupaba un papel secundario, no solo en el ámbito laboral, sino en la propia legislación civil, política y aún penal y, por supuesto en la vida social; su papel esencial en la sociedad era el dedicarse a las tareas reproductivas e incluso se incentivaba el abandono del trabajo una vez casada, para dedicarse al cuidado familiar con instituciones como la dote (indemnización por la extinción voluntaria del contrato de trabajo por parte de la mujer que se casaba -por supuesto con un hombre pues esa era la única pareja admitida-).

El impacto sobre esa legislación franquista de la aprobación de la Constitución y de la prohibición de la discriminación por razón de sexo introducida en el artículo 14 CE fue evidente.

Lo que ocurre es que inicialmente las medidas en torno a la discriminación fueron muy modestas y se centraron en una primera etapa en torno a las discriminaciones directas (Fabregat 2018-1, p. 753), incluso con tratamientos absolutamente insuficientes, aunque superadores de algunas aberraciones jurídicas como las normas que obligaban a la mujer a cesar en el trabajo al casarse (STC 7/1983, de 14 de febrero, seguida por otras muchas); así, por ejemplo, las numerosas sentencias en diversos tribunales sobre la dote (indemnización por cese -incluso voluntario- como consecuencia de contraer matrimonio, reconocida solo a la mujer), considerada inconstitucional pero sin que esa declaración fuese acompañada, por supuesto, de medidas que permitiesen a la mujer no abandonar el trabajo para dedicarse a tareas reproductivas y aún menos de medidas que incentivasen la corresponsabilidad; la consecuencia fue evidente, las mujeres en porcentajes amplios seguían abandonando el trabajo cuando se casaban pero sin compensación alguna por ello; no se incentivaba el abandono e incluso cuando alguna norma obligaba a él se declaraba esta como contraria a la CE, pero tampoco se evitaba a través de acciones sociales y medidas legales necesarias; por desgracia aún hoy ciertos empresarios siguen considerando molestas a las mujeres una vez han establecido relaciones de pareja y sobre todo cuando tienen hijos.

Es más, la interpretación acerca del doble mandato del artículo 14 que obligaba a los sujetos privados solamente a no discriminar pero poco más, dejaba sin atender la realidad histórica de muchos años de discriminación previa y mantenía los roles sociales sin alteración.

Es verdad que la tutela antidiscriminatoria se perfeccionaría sobe todo por influjo de la normativa y doctrina europea, extendiéndose también a las discriminaciones indirectas, 
especialmente a partir de la STC 145/1991, de 1 de julio, que, analizando un convenio que diferenciaba entre las retribuciones de los peones y las de las limpiadores -inferiores-, establecerá, introduciendo en el ámbito laboral el concepto de discriminación indirecta, que: "Ello permite ampliar y enriquecer la propia noción de discriminación, para incluir no sólo la noción de discriminación directa, o sea, un tratamiento diferenciado perjudicial en razón del sexo donde el sexo sea objeto de consideración directa, sino también la noción de discriminación indirecta, que incluye los tratamientos formalmente no discriminatorios de los que derivan, por las diferencias fácticas que tienen lugar entre trabajadores de diverso sexo, consecuencias desiguales perjudiciales por el impacto diferenciado y desfavorable que tratamientos formalmente iguales o tratamientos razonablemente desiguales tienen sobre los trabajadores de uno y de otro sexo a causa de la diferencia de sexo".

La recepción en nuestro derecho de la discriminación indirecta permitía así abordar discriminaciones grupales (Fabregat 2018-1, p. 753), pero en todo caso esa tutela antidiscriminatoria era insuficiente por varias razones, pero esencialmente porque era una tutela reactiva; como tal, reaccionaba frente a actos concretos, en relación con colectivos determinados o personas concretas, y lo hacía a posteriori, siempre tras haberse producido el acto discriminatorio.

La discriminación social quedaba así desprotegida y la necesaria tutela proactiva, necesaria, por un lado, para evitar a priori los actos discriminatorios y por otro, para garantizar la igualdad de oportunidades real entre mujeres y hombres, no se abordada y desde luego si algo se hacía era generalmente de modo aislado.

Es cierto que desde el primer Estatuto de los Trabajadores (ET) empezaron a aparecer preceptos que pretendían recordar la prohibición de discriminación en el ámbito laboral, preceptos que además resaltaban la necesaria igualdad en aspectos concretos y que se fueron perfeccionando; en la actualidad el ET está lleno de preceptos legales que así lo establecen en aspectos determinados, concretando la prohibición general de discriminación laboral contenida en los artículos 4.2.c) y 17 ET; así, por ejemplo, preceptos que reiteran la obligación de no discriminación en materias concretas podemos encontrar entre otros, en los artículos siguientes: el art. 22 ET en relación a la clasificación profesional; en relación a los ascensos el art. 24 ET; al respecto de la retribución (misma retribución ante trabajos de igual valor y registro salarial, arts. 9.3 y 28 ET); en relación con los despidos, declarando la nulidad en caso de incurrir en discriminación (arts. 53 y 55), etc.

Pero la tutela proactiva concretada por ejemplos en medidas como las de acción positiva tendentes a garantizar la igualdad de oportunidades, la igualdad material, combatiendo incluso discriminaciones sociales de profunda raigambre histórica se abrió paso muy lentamente en nuestra realidad e incluso en el ámbito internacional, como se analiza doctrinalmente (al respecto el magnífico estudio de Casas, 2019, pp. 13-54).

Incluso en sus primeros momentos, cuando se admitió por el TC se hizo, precisamente, para legalizar medidas de conciliación destinadas exclusivamente a la mujer (STC $128 / 1987$, de 16 de julio que contemplaba un complemento salarial destinada a compensar a las mujeres por el uso de guarderías) 
Esto, que en aquel momento era perfectamente comprensible y respondía a una realidad social, debió ir acompañada de medidas en orden a la corresponsabilidad porque de lo contrario se continuaba fomentando y perpetuando el rol social de la mujer como principal persona destinada a las tareas reproductivas. De hecho el TC reconocía en dicha sentencia la realidad social de que las mujeres tenían mayores problemas en el empleo laboral, incluso en el acceso al mismo y en su mantenimiento, por la tradición histórica que las relegaba a tareas de cuidado familiar, recordando que el artículo $14 \mathrm{CE}$ pero sobre todo el 9.2 CE obligan a los poderes públicos a remover esos obstáculos sociales, llamamiento al que ciertamente nuestro legislador ha tardado en responder.

Afortunadamente, hoy, la perspectiva limitada de las medidas de acción positiva se ha superado y, por ejemplo, el artículo 17.4 ET las legaliza plenamente y en muchos ámbitos estableciendo que: "Sin perjuicio de lo dispuesto en los apartados anteriores, la negociación colectiva podrá establecer medidas de acción positiva para favorecer el acceso de las mujeres a todas las profesiones. A tal efecto podrá establecer reservas y preferencias en las condiciones de contratación de modo que, en igualdad de condiciones de idoneidad, tengan preferencia para ser contratadas las personas del sexo menos representado en el grupo profesional de que se trate. Asimismo, la negociación colectiva podrá establecer este tipo de medidas en las condiciones de clasificación profesional, promoción y formación, de modo que, en igualdad de condiciones de idoneidad, tengan preferencia las personas del sexo menos representado para favorecer su acceso al grupo profesional o puesto de trabajo de que se trate."

Pero lo cierto es que hasta llegar a este artículo 17.4 ET y confiando en que se vaya concretando, el camino ha sido largo y en ese trayecto se sitúan la LOIEMH -en general- y los planes de Igualdad -en concreto en el ámbito laboral-.

En efecto, pese a los avances en la tutela antidiscriminatoria, pese a la admisión de medidas de acción positiva tendentes a garantizar la igualdad material, y pese a la numerosa cantidad de preceptos laborales que intentan garantizar la igualdad, lo cierto es que esta seguía sin existir; son evidentes las estadísticas que a la altura de 2007 -y aún hoy- demostraban que las tasas de actividad de la mujer eran inferiores a las de los hombres, que sus tasas de contratación temporal y a tiempo parcial eran por el contrario más elevadas que las de los hombres, que seguía existiendo una brecha salarial que incluso repercutía posteriormente en el ámbito de las prestaciones y pensiones de la seguridad social, que seguían siendo las mujeres las que en su mayor parte utilizaban las medidas destinadas a la conciliación de la vida laboral y familiar y que, incluso, no eran infrecuentes los supuestos en los que las personas empleadoras consideraban a la mujer como una empleada molesta sobre todo cuando era madre suponiendo que ella utilizaría las medidas de conciliación legalmente establecidas, todo ello entre otras muchas circunstancias que evidencian que la desigualdad pervive en el ámbito laboral (por todas Merino, 2009, pp. 15-16).

Junto al perfeccionamiento de los instrumentos legales laborales, lo cierto es que la LOIEMH nace del convencimiento de que solo una actuación integral que abordase el conjunto de situaciones que sitúan a la mujer en peores condiciones sociales podía contribuir a superar ese estado de cosas; la LOIEMH nace así con esa voluntad de tratamiento integral y, al margen de imperfecciones técnicas e insuficiencias, pretendía en el ámbito laboral que se 
abordasen también en él integralmente los problemas que perpetuaban la desigualdad laboral; para ello actuaba en varias direcciones pero destacaré esencialmente tres: en primer lugar perfeccionaba la tutela antidiscriminatoria; en segundo lugar mejoraba la legislación mediante correcciones en ciertos preceptos legales y, en tercer lugar y sobre todo, introducía la necesidad de negociar medidas de igualdad y, en ciertas empresas, planes de igualdad.

La LOIEHM pretendía ser así una ley integral y pretendía que en ese tratamiento integral, el protagonismo en el ámbito laboral lo asumiesen los planes de igualdad que debían ser los que en el ámbito concreto de cada empresa estableciesen el tratamiento laboral integral necesario para garantizar la igualdad, en el convencimiento de que sin esa concreción en el ámbito más reducido, los preceptos legales generales no conseguían desplegar totalmente su función igualitaria, pudiendo ser eficaces en su caso ante discriminaciones concretas pero no para garantizar una auténtica igualdad de oportunidades.

Lo que ocurre es que la LOIEHM fue muy modesta en la regulación de los planes de igualdad, lo que ha motivado su corrección posterior, en concreto en esta materia sobe todo en el RDL 6/2019, de 1 de marzo, cuyo impacto en la materia ha merecido interesantes estudios doctrinales (como, por ejemplo y por todos, Fabregat, 2019, o Aragón y Nieto, 2019). Sin duda es una corrección que puede ser más modesta que los numerosos proyectos de reforma legal que en años precedentes se habían producido y, por tanto, relativamente insuficiente en algunos aspectos, como alguna de la mejor doctrina ha expuesto (Ballester, 2019, pp. 15-18), y sin duda necesitaba de un cierto desarrollo reglamentario que clarifique ciertas cuestiones, pero que, en mi opinión y por lo que hace a los planes de igualdad, mejora considerablemente su régimen jurídico y responde mejor a su finalidad de tratamiento integral en el ámbito laboral, a ello me refiero a continuación.

\section{El plan de igualdad: su alcance, concepto y función}

Para entender el concepto y función y, por tanto, el alcance socio-jurídico del plan de igualdad, hay que partir de la definición legal del mismo y situarla en el marco de algunas ideas que ya he avanzado.

En efecto, el plan de igualdad ha de conseguir la igualdad real y actuar de modo integral.

Para ello es esencial entender que buena parte de las desigualdades proceden de los espacios de unilateralidad empresarial y que por tanto el plan se sitúa en el centro de una serie de conflictos, entre los que no es el menor el que se produce entre los poderes empresariales y el derecho a la igualdad material, a la igualdad de trato y oportunidades (analizando el supuesto concreto de la contratación alude a ese conflicto, por ejemplo, Olmo, 2013, p. 40).

También es esencial entender que, como se ha expuesto, la lucha por la igualdad es más compleja que un supuesto proteccionismo hacia la mujer; esto se ve muy claro en la adopción de medidas de conciliación; el objetivo actual no puede ser simplemente conciliar la vida laboral y familiar con la laboral, porque ello puede conducir, si no se acompaña 
de otras medidas más intensas, a perpetuar la división sexista de roles sociales y la dedicación de la mujer a las tareas reproductivas; por eso la igualdad lo que ha de conseguir es la corresponsabilidad, el que las tareas reproductivas se asuman por igual por hombres y mujeres, facilitando en igualdad de condiciones a todas las personas la conciliación de su vida personal, laboral y familiar (Lousada, 2008, pp. 7-8).

Por otro lado es esencial tener en cuenta que, ante esa complejidad, el plan no puede consistir en un conjunto de declaraciones genéricas o estandarizadas o, como ocurre a veces, en una reiteración de lo que ya existe en las normas legales, siquiera con leves retoques o mínimas mejoras.

En ese caso no se conseguiría lo que pretende la ley que es llevar al ámbito concreto de la realidad de la empresa la manera en que debe cumplirse la normativa general sobre igualdad entre mujeres y hombres, precisamente porque, como avancé, esa igualdad no se ha conseguido con la mera implementación de normas legales al respecto.

Ese es el sentido del concepto que la LOIEMH da al concepto del plan de igualdad al definirlo, en el artículo 46.1, como: "un conjunto ordenado de medidas, adoptadas después de realizar un diagnóstico de situación, tendentes a alcanzar en la empresa la igualdad de trato y de oportunidades entre mujeres y hombres y a eliminar la discriminación por razón de sexo. Los planes de igualdad fijarán los concretos objetivos de igualdad a alcanzar, las estrategias y prácticas a adoptar para su consecución, así como el establecimiento de sistemas eficaces de seguimiento y evaluación de los objetivos fijados."

Es más, si se tiene en cuenta la obligación general que para todas las empresas establece el artículo 45.1 LOIEHM, en el sentido de respetar la igualdad de trato y de oportunidades en el ámbito laboral y negociar y adoptar medidas dirigidas a evitar cualquier tipo de discriminación laboral entre mujeres y hombres, hay que considerar que el plan es la forma adecuada de que ciertas empresas, esencialmente las de más de 50 trabajadores, cumplan esa obligación.

Para cumplir con lo dispuesto y garantizar esa igualdad real, el plan no se elabora de cualquier forma, sino que debe contener un conjunto integral de actuaciones, que aborde transversalmente todos los aspectos de la relación laboral para detectar y corregir las desigualdades existentes y, como esta situación puede ser cambiante y ha de ser abordada en concreto, es fácil entender, como hace la doctrina y lo hizo desde el inicio de la LOIEHM, que solo cabe hablar de un plan de igualdad con el alcance que la norma pretende darle si este tiene esa transversalidad y se estructura como un conjunto de elementos estáticos y dinámicos, que respondan a la situación de presente cuando se elabora el plan pero que sean adaptables conforme a su evolución (Lousada,2014, pp. 7 y 8) y si contempla en forma suficiente las tres fases esenciales de cualquier plan de igualdad: diagnóstico, fijación de objetivos y medidas (acciones que conforman el plan) y control y seguimiento (por todos Fabregat 2018-1, pp. 756-757; Lousada, 2014, p. 8; Olmo, p. 38), todo ello además adoptado y negociado conforme a la realidad concreta y diferenciada de cada empresa.

Solo así el plan de igualdad responderá al concepto legal y, sobre todo, solo así será un instrumento útil para garantizar la igualdad real entre mujeres y hombres en la relación laboral. 
Sobre algunos aspectos centrales de este alcance del plan de igualdad me extenderé seguidamente.

\section{La expansión del ámbito aplicativo de los planes de igualdad}

Uno de los elementos más criticables de la redacción inicial de la LOIEHM era el escaso ámbito aplicativo de la obligación empresarial de establecer un plan de igualdad. En efecto solo las empresas de más de 250 trabajadores quedaban obligadas a hacerlo. En definitiva, solo en el marco de la gran empresa se establecía esa obligación (Fabregat, 2019 , p. 24) lo que limitaba mucho el impacto de la medida, sin que por otra parte se haya cumplido en su totalidad, siquiera en ese ámbito.

Podía entenderse esa limitación aplicativa por la novedad, por el hecho de que ciertamente en los demás casos también había obligación de evitar la discriminación y adoptar medidas, en su caso negociadas, que garantizasen la igualdad entre mujeres y hombres, pero ciertamente se disminuía mucho el impacto de la medida y eso pese a que se preveía la posibilidad de que otras empresas voluntariamente adoptasen un plan de igualdad, que la negociación colectiva lo impusiese o que la autoridad laboral acordase en un procedimiento sancionador la sustitución de las sanciones accesorias por la elaboración y aplicación de dicho plan. Además, es cierto, que en el ámbito del sector público, todas las Administraciones Públicas, con independencia del número de personas a las que empleasen, estaban y están obligadas a establecer negociadamente un plan de igualdad (Disposición adicional séptima, 2, del Estatuto Básico del Empleado Público, cuyo texto refundido fue aprobado por RD Legislativo 5/2015, de 30 de octubre), sin que tampoco en este ámbito se haya cumplido plenamente la obligación, resultando incluso paradójico que este año 2020 es cuando se haya aprobado el primer plan de igualdad de las propias Cortes, órgano que aprobó la LOIEMH trece años antes.

La limitación de la obligación era evidente pues, conforme a los datos del Ministerio de Trabajo, Migraciones y Seguridad Social, en enero de 2019, las empresas de más de 250 trabajadores eran solamente 4.700, mientras que las de menos que contaban al menos con un asalariado eran 1.322.261 empresas. Es cierto que en número de trabajadores afectados el impacto era mayor pues esas 4.700 empresas de más de 250 trabajadores, daban empleo a 5.352.361 personas, mientras que las de menos de 250 trabajadores, daban empleo a 8.416.423 personas. Es decir, si el número de empresas afectadas por la obligación era muy pequeño, por lo menos el de personas trabajadoras afectadas alcanzaba casi un $40 \%$ del conjunto.

Por eso, la extensión de la obligación a un número superior de empresas, al amparo del RDL 6/2019, ha sido calificada como una de las medidas interesantes de la reforma (Ballester, 2019, p. 20), aunque ciertamente, según esas mismas cifras y en esa misma fecha, la expansión de la obligación alcanzaría solo a las 24.508 empresas con un número de personas trabajadoras comprendidas entre 50 y 249, lo que en términos de personas 
empleadas sí que es, por el contrario, muy significativo porque supone incorporar al ámbito de la medida a un total de 2.400 .131 personas empleadas más.

Aun así quedarán todavía un total de unos 6.000 .000 de personas trabajadoras sin que sus empresas estén obligadas a acogerse a un plan de igualdad.

Es posible que estas cifras deban corregirse algo pues, como dije, además de las que lo estén por el elemento cuantitativo, pueden existir otras empresas obligadas y todas las Administraciones lo están, pero las cifras expuestas dan buena cuenta de la expansión en el ámbito aplicativo de la reforma legal.

Por otro lado, la expansión del ámbito subjetivo de la obligación se aplica progresivamente; así en el primer año posterior a la publicación del RDL 6/2019 (producida el 7 de marzo de 2019), la obligación se extendía a las empresas de más de 150 personas trabajadoras; las de 100 a 150 contaban con dos años desde esa fecha para la aplicación de la obligación y tres años las que contasen entre 50 y 100 personas trabajadoras (Disposición transitoria decima segunda LOIEHM).

Una vez implantada totalmente la obligación, todas las empresas de más de 50 personas trabajadoras deberán contar con un plan de igualdad, y entonces la mayoría de las personas trabajadoras quedarán acogidas a alguno de estos planes; sin duda, si la obligación se cumple realmente y no de manera meramente formal, puede producirse un avance significativo en la igualdad en el ámbito laboral.

En todo caso, la regulación plantea dos problemas concretos: cómo medir el número de trabajadores y el ámbito concreto en el que debe negociarse e implantarse el plan de igualdad.

En cuanto al primer problema, parece claro que la norma legal no introduce matices y por tanto deben computarse todos los trabajadores que formen parte de la plantilla de la empresa, tanto trabajen a tiempo completo como a tiempo parcial y tanto sean fijos, como fijos discontinuos, como temporales, resultando igualmente indiferente que se sujeten a relación laboral ordinaria o especial, y tanto trabajen presencialmente como a distancia. Es más, no hay fundamento legal que permita utilizar reglas de cómputo teórico similares a las que se aplican en materia de elecciones a representantes de los trabajadores, pero no a otros efectos en los que, al igual que se sostiene que debe hacerse aquí, se computan todas las personas trabajadoras con independencia del vínculo contractual - por ejemplo, art. 1.1. del Reglamento de los procedimientos de despido colectivo y de suspensión de contratos y reducción de jornada, aprobado por RD1483/2012, de 29 de octubre.

Incluso la obligación del plan de igualdad una vez adoptado, se mantiene aunque existan fluctuaciones temporales del número de personas empleadas que en algún momento concreto puedan hacer descender el número por debajo de 50 personas empleadas, solución que defiende la mejor doctrina (Fabregat, 2019, pp. 29-31) y que me parece ciertamente la aplicable, pues el plan de igualdad no puede ser una medida aplicada a ratos o temporadas en función de la variabilidad de la plantilla, además de que el número de trabajadores inicial que motivó la obligación del plan es el relevante a efectos de la misma; cuestión distinta sería una disminución permanente de plantilla que llevaría a que no 
existiese la obligación de volver a negociar un plan de igualdad pero, como la obligación de garantizar la igualdad sigue vigente para cualquier empresa y el plan lo único que hace es concretarla en ese ámbito, es casi seguro que las medidas del plan seguirían siendo aplicables, al menos en su contenido material y, desde luego y como dije, el plan, una vez negociado, debe mantenerse en vigor el tiempo acordado sin ser alterado en su vigencia por oscilaciones de plantilla.

En cuanto al ámbito de aplicación del plan, la norma legal da respuesta clara (art. 46.3 LOIEMH). En efecto, el precepto concreta que en las empresas con diversos centros de trabajo el plan debe afectar a toda la empresa sin perjuicio de que se contemplen medidas específicas para determinados centros. Se está rechazando, pues, la negociación y consiguiente aplicación de planes en ámbito infraempresarial, posiblemente para evitar que en una empresa se llegase a contar con centros con plan y otros sin él y para garantizar un tratamiento integral en toda la empresa de las cuestiones relativas a la igualdad; lo anterior no implica, como la norma aclara, que no deban tenerse en cuenta las situaciones específicas y diferenciadas de ciertos centros de trabajo, e incluso dentro de un centro de secciones o departamentos determinados; es más, las mismas deben contemplarse en atención a lo que se desprenda del diagnóstico de situación que puede evidenciar problemas específicos en materia de igualdad y requerir por tanto medidas apropiadas, incluso si así se considera necesario con seguimiento específico, pudiendo existir órganos articulados para hacer seguimientos más directos en cada centro, coordinados con el seguimiento general que siempre debe producirse en la empresa en relación con todo el plan.

La exigencia de que el plan ofrezca respuesta concreta a los problemas reales, implica contemplar esas situaciones específicas que puedan darse en centros concretos, pero no permite, desde luego, una negociación diferenciada por centros que podría ser perjudicial para la finalidad pretendida de conseguir un tratamiento integral y transversal.

Por eso mismo, la negociación del plan debe producirse en el ámbito de la empresa y no en el ámbito sectorial, o en cualquier otro supraempresarial, sin perjuicio de que quepa una cierta articulación en la medida y la negociación colectiva sectorial y supraempresarial pueda fijar criterios generales y ofrecer pautas a la negociación en la empresa, conforme al art. 85.2.b) ET (Fabregat 2018-1, p.747), como también extender la obligación de contar con plan a más empresas e incluso ofrecer soluciones para problemas concretos como, por ejemplo, la falta de interlocutores negociales en algunas empresas o establecer sistemas de solución de los desacuerdos, aunque la negociación colectiva en la materia no había sido especialmente amplia e imaginativa, sobre todo en lo referente a acciones positivas antes de la ley (Cabeza, 2007, p. 36), ni tampoco parece que haya progresado demasiado aunque sí empiezan a aparecer experiencias al respecto, remitiéndome en todo caso a los estudios que sobre la materia se han efectuado doctrinalmente (por ejemplo y por todos, Cabeza, 2018 y Sánchez (dir.), 2016; Domínguez, 2018, y, en lo referente al sector público, García (dir.), 2019, especialmente pp. 201-244). 


\section{Contenido y características esenciales del plan de igualdad}

El plan de igualdad debe tener un contenido y responder a unas características que lo definen en atención a la función que debe cumplir.

Al respecto considero esencial resaltar cuanto sigue.

\subsection{Carácter integral}

Como ya expuse, el plan de igualdad es un conjunto de medidas que pretenden dar una respuesta integral a las situaciones de desigualdad que en cada empresa existan. Ese carácter de respuesta integral exige que las medidas sean lo más concretas posibles y, por tanto, se hace esencial el estudio de la situación específica de cada empresa -la fase de diagnóstico a la que luego me referiré-. Como se ha expuesto, aunque adoptase formalmente la estructura de un plan de igualdad, no lo sería, ni un conjunto de medidas genéricas alejadas de la situación concreta de una empresa, ni la reproducción de medidas basadas en realidades distintas a la de la empresa en cuestión, como por ejemplo las adoptadas en planes de otras entidades (Lousada, 2014, p. 8); en definitiva, el plan debe adoptar medidas específicas, previstas ad hoc para la realidad de cada empresa (Luján y Selma, 2016, p. 463) y su carácter integral viene a exigir que se cumplan dos circunstancias: la primera que el plan aborde todos los aspectos de la relación laboral para erradicar las consecuencias de cualquier tipo de discriminación que pudiera existir -directa, indirecta o social-, pero también para prevenir en cuanto sea posible que las mismas no se produzcan (Fabregat, 2019, p. 17); la segunda, que el plan preste la debida atención a las tres fases dinámicas en que se estructura (diagnóstico, adopción de medidas, control y seguimiento), especialmente esta última fase es esencial y debe existir el convencimiento de que el logro de la igualdad, la obligatoriedad del plan de igualdad, no es una obligación que se agota en un solo acto y produce un conjunto de medidas invariable y petrificado, sino que es un deber de cumplimiento sucesivo (Luján y Selma, 2016, p. 466), que obliga a evaluar el grado en el que se van alcanzando los objetivos pretendidos y, en función del mismo, revisar y adaptar las medidas adoptadas; en definitiva el plan de igualdad debe ser dinámico, variable, cambiante como lo es la realidad a la que intenta hacer frente (Fabregat, 2019, p. 99).

Solo cumpliendo estas exigencias puede pensarse que se está ante un conjunto de medidas que aborden en su integridad la desigualdad en la empresa, y solo así se garantiza, pues, el carácter integral del plan.

\subsection{Diagnóstico de situación y plan de igualdad}

Expuesto lo anterior, puede entenderse la importancia que tiene la primera fase del plan, el estudio de la realidad de la empresa sobre la que va a proyectarse, la fase de diagnóstico. 
Pese a esa importancia, no era infrecuente que, antes de la reforma introducida por el RDL 6/2019, el diagnóstico no se negociase y se elaborase por la propia empresa (Lousada, 2014, p. 11), en su caso mediante gabinetes técnicos y similares; falta de negociación del diagnóstico que se consideraba que no conducía de por sí a la nulidad del plan (como resolvió la sentencia de la Audiencia Nacional (SAN) (social) 12/11/2019, proc. 195/2019).

Precisamente esa falta de análisis concreto conducía a dos errores muy frecuentes, a los que ya me he referido y que aparecían en muchos planes: limitarse a declaraciones tan genéricas que se quedaban en el mero plano de lo teórico, de la abstracción; o, en segundo lugar, limitarse a reproducir derechos legales ya reconocidos, especialmente vinculados a medidas de conciliación (Luján y Selma, 2016, p. 456).

Esa relativa devaluación del diagnóstico, que impactaba claramente en la eficacia del propio contenido del plan, ha sido corregida por el RDL 6/2019 que mantiene a este como fase previa ineludible pero dejando claro, lo que en mi opinión ya debía haberse entendido siempre así, que el mismo debe ser negociado y que debe responder al contenido integral que se espera del análisis, estableciendo en consecuencia un contenido mínimo obligatorio (Fabregat Monfort, 2019,p. 48), pues la LOIEMH señala que el diagnóstico contendrá al menos las siguientes materias: a) Proceso de selección y contratación. b) Clasificación profesional. c) Formación. d) Promoción profesional. e) Condiciones de trabajo, incluida la auditoría salarial entre mujeres y hombres. f) Ejercicio corresponsable de los derechos de la vida personal, familiar y laboral. g) Infrarrepresentación femenina. h) Retribuciones. i) Prevención del acoso sexual y por razón de sexo.

Reitera además el art. 46.2 LOIEMH que la negociación del diagnóstico se produce en la propia comisión negociadora del plan de igualdad, y establece la obligación empresarial de suministrar toda la información necesaria al respecto, incluidos los datos del registro salarial a que se refiere el art. 28.2 ET.

El cambio legal es sustancial pues, donde antes existía un contenido difuso del diagnóstico y un listado ejemplificativo de materias a incluir en el plan de igualdad, tras el RDL 6/2019 existe un contenido claro, concreto y obligatorio del diagnóstico que, como se verá, lógicamente tendrá su reflejo en el contenido del plan, reforzando así esta fase esencial para que el plan descienda al terreno de lo concreto, de la realidad empresarial, y aborde así con la seriedad debida la erradicación de cualquier situación de desigualdad que se pueda detectar.

Además, como puede verse, y confirmando lo que avancé, el listado de materias incide en ámbitos en los que el poder empresarial es fuerte y, muchas veces, poco limitado, como en el acceso al empleo o en la promoción -especialmente a puestos directivos-; la norma legal es consciente de que la unilateralidad empresarial es fuente en no pocas ocasiones de desigualdades, por ello genera un instrumento que lleva a limitar y contractualizar esos poderes empresariales, siquiera a efectos igualatorios.

Por otro lado, la norma reacciona frente a una confusión frecuente; así se reitera, siquiera indirectamente, que conciliación no es sinónimo de igualdad, sino que lo que conduce a la igualdad es la corresponsabilidad, el reparto de las tareas reproductivas entre 
mujeres y hombres y, por eso, el contenido del diagnóstico en la materia no es esencialmente el análisis de las medidas de conciliación, sino el análisis del uso corresponsable de las mismas. Sin despreciar la importancia de las medidas de conciliación, como se ha expuesto, la igualdad lo que esencialmente exige en esta materia es adoptar medidas para impedir que persista la división sexista de las responsabilidades familiares (Casas, 2019, p. 46).

Son, entre otros, claros mensajes dirigidos a quienes deben negociar el plan de igualdad.

La reforma legal ha dotado de instrumentos precisos a las partes negociadoras para que puedan combatir la desigualdad, del uso que hagan de esas herramientas depende en buena medida el éxito de la reforma legal y la consecución de los objetivos igualitarios que se pretenden.

Es más, conviene no olvidar que, al margen de que las discriminaciones constituyan infracción laboral muy grave, la propia transgresión de las normas que rigen la elaboración de los planes de igualdad y, por tanto, también la falta de colaboración o incumplimiento de la empresa en la elaboración del diagnóstico puede ser constitutiva de una infracción grave al amparo del artículo 7.13 de la Ley de Infracciones y Sanciones en el Orden Social, aprobada por Real Decreto Legislativo 5/2000, de 4 de agosto.

\subsection{El carácter negociado del plan de igualdad}

Sin despreciar la importancia que puedan tener medidas establecidas unilateralmente por las empresas, incluso en el marco de las iniciativas de responsabilidad social (como analiza, entre otras, Merino, 2009, pp. 9-82), lo cierto es que lo esencial de las medidas igualatorias en las empresas obligadas a contar con plan de igualdad han de venir de la negociación colectiva, porque el plan, desde su inicio, como se ha visto en relación con la fase de diagnóstico, es un instrumento negociado.

Así lo concreta claramente la ley y ya se analizó además que establece que el ámbito natural de negociación es la empresa.

Incluso así lo ha interpretado la jurisprudencia y doctrina judicial (un buen análisis al respecto, por ejemplo, en Poquet, 2019, pp. 89-97 y en general y en excelente análisis de la interpretación judicial acerca de los planes de igualdad y no solo sobre este aspecto, en Monereo y Guindo, 2018, pp. 565-579).

Ahora bien, la exigencia de negociación plantea varios problemas jurídicos, esencialmente los siguientes: 1) ¿es exigible siempre ese carácter negociado? 2) determinar los sujetos legitimados para negociar; 3) ¿qué ocurre si no hay sujetos legitimados para negociar? y 4) ¿cuál es la naturaleza jurídica del plan?

Analicemos estos problemas esenciales

1) Como regla general el plan de igualdad es un instrumento negociado; lo es necesariamente en el ámbito público (Disposición adicional séptima. 2. EBEP) y esa es también 
la regla general en el ámbito privado, pero en este cabe diferenciar supuestos en los que la obligación no es tan clara (Fabregat, 2018-2, pp. 378-381).

En efecto, para las empresas obligadas a contar con plan de igualdad en atención al número de personas empleadas, la norma legal reitera que el plan debe ser negociado (45.2 LOIEMH), remitiendo al respecto a lo que disponga la legislación laboral, que reconduce a la negociación de convenios (art. 85.2 ET), reiterando posteriormente, como se analizó, la obligación de negociar el diagnóstico y aunque es cierto que hay una remisión a un desarrollo reglamentario de bastantes aspectos (46.6 LOIEMH), no es menos cierto que la obligación de negociar es clara y no necesita mayor desarrollo y que en las restantes materia sujetas a desarrollo reglamentario la norma legal permite ya su aplicación, por contar con una regulación suficiente, en ausencia del mismo.

Así pues, en estas empresas la implantación del plan sin acuerdo conduce a su nulidad, como se ha resuelto jurisprudencialmente (STS (social) 13/09/2018, Recurso n. 213/2017 y STS (social) 09/05/2017, Recurso n. 85/2016; en el mismo sentido, por ejemplo, SAN (social) 10/12/2019, proc. 163/2019), salvo supuestos excepcionales de bloqueo de la negociación imputable exclusivamente a la parte no empresarial y siempre que el empleador haya agotado todas las posibilidades, incluso extrajudiciales, de solución del bloqueo.

Ahora bien, junto a las empresas obligadas cuantitativamente en virtud de la norma legal, se permite que los convenios amplíen a otras la obligación, remitiendo en este caso a los términos que establezcan esos convenios (art. 45.3 LOIEMH). Lo normal y deseable es que estos impongan la obligación de negociar el plan pero la ley no lo establece literalmente; pese a ello, creo, aunque es un supuesto dudoso, que en este caso la regla general debe mantenerse, pues la previsión de la posibilidad de no negociar no se contempla tampoco expresamente y sí se hace en los dos supuestos que a continuación indicaré. Esa diferencia de regulación hace entender, en mi opinión, que la norma legal no permite al convenio excepcionar el carácter negociado del plan; tendrá la opción de extender o no a más empresas la obligación de contar con plan de igualdad, pero no la de alterar sus características esenciales, entre las que se encuentra el carácter negociado que en estos momentos se establece como sustancial en el diagnóstico, y que solo podría omitirse en los casos en que la propia ley lo admite expresamente remitiéndose a la alternativa de consultas.

Esto enlaza, además, con el artículo 45.1 LOIEMH, que establece la obligación general de negociar, y en su caso acordar, las medidas en materia de igualdad.

Como dije, es un supuesto que plantea dudas, pues cabe desde luego la opinión contraria que incluso parece desprenderse de alguna sentencia como STS (social) 13/09/2018, Recurso n. 213/2017, y que es defendible también en una interpretación extensiva de la remisión que la norma hace en estos supuestos a los términos del convenio, aunque en mi opinión esa remisión no debería interpretarse en un sentido tan amplio que permitiese cuestionar las bases conceptuales del plan de igualdad, que lo identifican como un instrumento adaptado a la realidad de la empresa, integral, dinámico y participado (negociado y en su caso acordado). Estos serían límites que el convenio debería respetar, sería bueno que siempre quedase claro y, desde luego, y si no se comparte esta interpretación, cuando 
menos el plan debería ser consultado, exigencia que se hace incluso para los supuestos en que ni siquiera es obligada su implantación como se verá a continuación.

Por el contrario, y como acabo de avanzar, en otros dos supuestos la norma legal sí contempla la posibilidad de que no exista negociación sino simplemente consulta. Uno es el de las empresas que no estén obligadas a contar con plan de igualdad pero que voluntariamente deseen implantarlo -lo que en muchos casos vendrá incentivado por el acogimiento a posibilidades de subvenciones y ayudas o contrataciones públicas-. En este caso la norma legal dice que el plan se implantará por la empresa previa consulta a la representación legal de las personas empleadas (art. 45.5 LOIEMH), lo que excluye claramente la obligación de negociar el plan, aunque esto será siempre posible de forma voluntaria.

El segundo supuesto es el de las empresas obligadas por acuerdo de la autoridad laboral, en un procedimiento sancionador para sustituir las sanciones accesorias por la elaboración y aplicación de dicho plan, en los términos que se fijen en el indicado acuerdo y en cuyo caso la norma señala que el plan deberá ser negociado o consultado con la representación legal de las personas que trabajen en la empresa. La interpretación lógica del precepto conduce a que en este caso existe obligación de negociar el plan pero que, si en dicha negociación no se alcanza acuerdo, la empresa pueda implantar unilateralmente el plan, bastando la consulta, y sujeto, eso sí, a que la propia autoridad laboral compruebe que se ajusta a los términos del acuerdo administrativo que obligó a la empresa.

2) En cuanto a los sujetos legitimados para negociar, la solución es clara, son los llamados a negociar en la Administración Pública en las correspondientes mesas de composición sindicalizada, y en el ámbito privado los legitimados para negociar en el ámbito de la empresa el convenio (art. 85.2 ET), lo que conforme al ET da preferencia a la negociación con las secciones sindicales en los términos del artículo 87.1 ET, e incluso directamente con las organizaciones sindicales por analogía con lo resuelto para la negociación de convenios, pero no impide en ese ámbito laboral que también estén legitimadas las representaciones unitarias, si bien lógicamente, en las empresas con varios centros y salvo que cuenten con comité intercentros - que es el auténtico comité de empresa en esas empresas - con capacidad negocial (art. 63.3 ET), como el ámbito de las representaciones es el del centro en el que son elegidas pero el ámbito del plan es toda la empresa, lo más práctico y seguro en aras a respetar las reglas negociales y la consiguiente correspondencia de ámbitos entre la negociación y la representación, es que acaben negociando las secciones sindicales o los sindicatos.

Desde luego lo que no cabe es sustituir a los sujetos legitimados por otro tipo de representaciones, comisiones ad hoc o cualquier otra parecida, como con claridad se resuelve en la jurisprudencia (STS (social) 14/02/2017, Recurso n. 104/2016), ni omitir la presencia de cualquier sujeto legitimado para negociar (STS (social) 11/03/2014, Recurso n. $77 / 2013)$.

3) Determinar lo que ocurre cuando no hay representaciones legitimadas para negociar en nombre de las personas empleadas es complejo. 
Por un lado es evidente que no puede exigirse a la empresa cumplir con una obligación imposible como sería negociar con una representación inexistente; también es claro que la norma legal no ha previsto soluciones alternativas como comisiones negociadoras ad hoc y similares; finalmente también parece evidente que esta cuestión es una de las que debería ser abordada en la negociación sectorial, por lo que si en la misma se establecen soluciones al problema a ellas habrá que estar.

Ahora bien, si no hay reglas ni siquiera convencionales al respecto, solo caben dos soluciones: entender amortizada la obligación de negociar o entender que debe negociarse necesariamente con algún sujeto representativo como, seguramente, los sindicatos representativos en el ámbito sectorial al que pertenezca la empresa, porque lo que desde luego no parece admisible es que esa circunstancia condujese a excepcionar la propia obligación de contar con plan de igualdad.

Lo deseable es que existan soluciones convenidas, posiblemente en los términos que he dado como ejemplo, es decir negociando con las organizaciones sindicales directamente, pero en ausencia de reglas negociales es dudoso, aunque deseable, que pueda imponerse a la empresa la negociación del plan con sujetos diferentes a los previstos normativamente (al respecto STS (social) 13/09/2018, Recurso n. 213/2017), pareciendo que en ese caso la obligación se cumpliría con la implantación unilateral sujeta a comprobación judicial o administrativa de que el plan se ajusta a las exigencias legales, y con obligación de negociación tan pronto existiese representación legitimada para ello. En todo caso esta solución altera la delimitación conceptual del plan como instrumento participado y, por ello, deben explorarse las posibles interpretaciones que puedan conducir a una negociación sindicalizada en ausencia de otras representaciones en la empresa, aunque, como he expuesto, son complejas de sostener.

4) En cuanto a la naturaleza jurídica del plan, creo que hay que diferenciar dos supuestos; cuando el plan no es negociado, supuesto residual como ya dije, no puede tener otra naturaleza que la de un acto unilateral de la empresa y, por tanto, estamos ante un compromiso de la empresa, si se quiere ante una decisión empresarial de efectos colectivos que le vincula y que debe ceder, en su caso, ante cualquier otro instrumento negociado que resulte mejor para las personas que trabajan en la misma.

Por el contrario, cuando el plan es negociado en los términos establecidos en la ley, me parece que estamos ante un instrumento convencional, de eficacia general y normativa, tanto se contenga en el convenio de empresa, en cuyo caso esa naturaleza es evidente, como cuando se produzca como una negociación diferenciada, en cuyo caso estaríamos ante un convenio de contenido limitado pero no por ello menos eficaz, ni de naturaleza y régimen jurídico diferente al de cualquier otro convenio.

Desde esta perspectiva, en este caso, como ya avancé, las reglas de legitimación negocial son las propias de la negociación colectiva y la eficacia, duración y vigencia y problemas de sucesión normativa y concurrencia deben regirse por las reglas propias de la negociación estatutaria, esencialmente establecidas en los artículos 82, 84 y 86 ET, lo que 
como solución general me parece lógico, aunque puede plantear algún problema de aplicación práctica en el que no me puedo detener en esta aproximación.

\subsection{Contenido variable}

Como indiqué, uno de los importantes cambios introducidos en el RDL 6/2019 fue establecer un contenido mínimo obligatorio para el diagnóstico, mientras que el contenido del plan queda más indeterminado, estableciéndose que contendrá los concretos objetivos de igualdad a alcanzar, las estrategias y prácticas a adoptar para su consecución, así como el establecimiento de sistemas eficaces de seguimiento y evaluación de los objetivos fijados.

Posiblemente, al determinar el contenido del diagnóstico, la norma legal pretendía evitar incorrecciones muy frecuentes en los planes de igualdad que hasta el momento tenían, en atención a las amplias posibilidades de incorporar materias con diversas regulaciones, contenidos muy variados, que han sido objeto de interesantes estudios doctrinales en general o en relación con contenidos particulares (por ejemplo, en relación con contenidos en general puede verse Alfonso (coord.) 2013, Olmo, 2013 y, entre otros aspectos por su análisis sobre errores frecuentes, Selma, 2017; y en relación con contenidos concretos, por ejemplo, Saldaña, 2011 y 2013; o incluso elaborando interesantes guías para la negociación como, entre otras, la de Romero, 2017, o evidenciando como las insuficiencias y deficiencias de contenido afectan seriamente a la propia eficacia del instrumento, como analiza Grau, 2020, versión no impresa).

Esa amplitud de posibles contenidos conducía, como acabo de exponer, a notables insuficiencias en algunos planes, bien porque se limitaban en general a reiterar regulaciones legales o a establecer cláusulas genéricas o estandarizadas sin relación con la realidad empresarial, o incluso porque, con buena intención pero equivocadamente, se limitaban a tratar ciertas temas, normalmente conciliación, medidas para víctimas de violencia de género y procedimientos en materia de acoso (Grau, 2014, pp. 263-286), considerando que esos eran los temas asociados a la igualdad, pero sin abordar cuestiones más transversales y esenciales como acceso, clasificación y promoción, retribuciones, etc., lo que hacía que la desigualdad perviviese.

La norma ha pretendido invertir los términos y obligar al análisis transversal al que me referí, de tal modo que sean los resultados del mismo, del diagnóstico, los que condicionen el contenido del plan.

Lo anterior introduce la duda de si todas las materias que deben ser objeto del diagnóstico deben encontrar reflejo en el plan; en mi opinión el diagnóstico fijará la intensidad de las medidas a adoptar, los objetivos a conseguir y los indicadores de seguimiento, pero todas las materias objeto de diagnóstico deben encontrar reflejo en el plan, siquiera y como se ha expuesto doctrinalmente para reflejar que en alguna de ellas no se aprecia discriminación ni necesidad de actuación, lo que incluso facilita el control por la autoridad laboral ante el obligado registro de los planes al amparo del artículo 46.4 y 5 LOIEMH (Fabregat, 2019, pp. 72-73). 
Realmente lo anterior debe ser así, pues es lo único que se ajusta al carácter dinámico del plan; en efecto, que en una situación estática, en el presente, no se detecten necesidades de actuación, no impide que la situación evolucione y requiera en el futuro actuaciones igualatorias; desde esta perspectiva dinámica, todas las materias que como mínimo deben diagnosticarse deben ser objeto de seguimiento para comprobar su evolución y que, en su caso, siguen sin evidenciar la necesidad de medidas en orden a garantizar o restablecer la igualdad.

En mi opinión, pues, el contenido mínimo del diagnóstico es, a su vez, contenido mínimo del plan y objeto de su seguimiento, aunque no necesariamente deberán adoptarse medidas en todo ese contenido, sino solo en aquellas materias en que resulten necesarias y con la intensidad que los resultados de la evaluación, del diagnóstico, hagan precisa.

En cuanto a otros aspectos del contenido me remito a los estudios citados y a los que he ido exponiendo en el texto.

\subsection{Naturaleza temporal y dinámica}

Finalmente, en este breve repaso a algunas cuestiones que me han parecido esenciales para situar el alcance socio-jurídico de los planes de igualdad, quiero reiterar que las medidas del plan de igualdad, y en realidad todo el plan, tienen un alcance de temporalidad que les resulta esencial en cuanto que están dirigidas a modificar la realidad sobre la que actúan y, por tanto, deben evolucionar en atención a la evolución de la propia realidad que pretenden transformar; así lo ha entendido toda la doctrina que se ha referido al dinamismo de los planes de igualdad (por todos Fabregat, 2019, pp. 99-102; Lousada, 2014, pp. 7-8; Olmo, 2013, p. 39; Grau, 2020 -versión no impresa-, etc.) y solamente así se garantiza la necesaria eficacia en materia de igualdad de plan.

Por lo expuesto, corresponde a las partes que negocian el plan establecer su dimensión temporal, pero no solo en términos de duración del mismo, sino también en cuanto al calendario de adopción de las medidas previstas, periodos de revisión de la situación en cuanto a los objetivos fijados e indicadores para medir el cumplimiento, órganos destinados al control y seguimiento y capacidad de los mismos para revisar, en su caso, las medidas adoptadas, teniendo en cuenta que, si estos órganos tienen capacidad para renegociar o alterar el plan, deberán respetar en su composición las reglas de legitimación para la negociación que en cada caso proceda aplicar, pudiendo en caso contrario estar compuesta solamente por quienes firmaron el acuerdo o plan, pues las funciones serán únicamente la interpretación, aplicación, seguimiento y en su caos propuestas pero nunca alterar la regulación pactada (como ejemplo de esta doctrina general, puede verse STS (social) 30/05/2018, rec. 147/2017).

En todo caso la capacidad de revisar modificando el plan no se supone ni se la puede atribuir a sí mismo el órgano de seguimiento (SAN (social) 26/06/2019, proc. 101/2019), pues la decisión al respecto corresponde a los negociadores del plan. 
Solo con una correcta regulación de estos aspectos dinámicos se cumple con las previsiones legales, en orden al establecimiento de sistemas eficaces de seguimiento y evaluación de los objetivos fijados en el plan (art, 46.1 LOIEMH), y encuentran sentido las obligaciones empresariales de información sobre el grado de cumplimiento de los objetivos (art. 47 LOIEMH).

La variedad de sistemas y órganos de seguimiento puede ser muy amplia y de hecho la propia ley alude a las comisiones paritarias de los convenios si se les atribuye esa función, pero nada impide la existencia de comisiones específicas o de otro tipo de órganos con funciones de seguimiento, con el limite lógico de que el seguimiento debe hacerse con participación de las partes que han negociado el plan y no puede quedar a la unilateralidad empresarial, lo que desvirtuaría totalmente el sentido del mismo y del propio plan como instrumento esencialmente negociado.

\section{Unas breves conclusiones}

Con este trabajo he pretendido exponer algunos de los elementos esenciales que pueden resaltarse en la actual configuración jurídica de los planes de igualdad, especialmente en cuanto a su contexto y contenido, pues otras cuestiones se abordan en los restantes estudios expuestos en la misma jornada que el que da origen a este trabajo, y son objeto, también, de publicación en esta misma revista.

Especialmente he pretendido resaltar la necesidad de valorar, regular e interpretar los planes de igualdad desde la cláusula de Estado Social y del valor esencial que en el mismo tiene la igualdad real y material. Es más, solo desde esa vinculación cabe entender la propia regulación en materia de igualdad efectiva entre mujeres y hombres, sus modificaciones y, por supuesto, la propia evolución en la regulación jurídica de los planes de igualdad, culminada por el momento en el RDL 6/2019 que, con acierto, viene a resaltar aspectos como el contenido del diagnostico o el carácter negociado del mismo, aspectos que resultan esenciales para la propia eficacia real del plan de igualdad como instrumento transversal que garantice la igualdad real en la empresa.

Quiero finalizar este breve repaso con mi convencimiento de que, bien negociados, los planes de igualdad pueden suponer una herramienta muy útil (Aragón y Nieto, 2019, p. 323), un avance hacia la igualdad real entre mujeres y hombres en el ámbito laboral, objetivo que, como dije, es irrenunciable en un Estado Social y que ya lleva demasiado tiempo sin conseguirse plenamente.

\section{Bibliografía}

ALFONSO MELLADO CARLOS L. (coord.) (2013) y Cordero Gordillo, Vanessa; Fabregat Monfort, Gemma; Moreno Solana, Amanda y Morillo Balado, Rosario Observatorio de la negociación colectiva. La negociación en materia de planes de igualdad: estudio analítico, Madrid, Ediciones Cinca. 
ARAGÓN GÓMEZ, CRISTINA y NIETO ROJAS, PATRICIA (2019) “El impulso a los planes de igualdad en el RD-Ley 6/2019”, pp. 321-374, Tiempo de reformas, De la Puebla Pinilla, Ana y Mercader Uguina, Jesús (dirs.), Valencia, Tirant Lo Blanch.

BALLESTER PASTOR, MARÍA AMPARO (2019) “El RDL 6/2019 para la garantía de la igualdad de trato y de oportunidades entre mujeres y hombres en el empleo y la ocupación: Dios y el diablo en la tierra del sol", Femeris, Vol. 4, No. 2, pp. 14-38.

CABEZA PEREIRO, JAIME (2007) "Los planes de igualdad y la negociación colectiva", $A e$ qualitas, n. 21, 2007, pp. 35-46.

CABEZA PEREIRO, JAIME (2018) "Igualdad efectiva de mujeres y hombres en la negociación colectiva”, pp. 683-702, El derecho de igualdad efectiva de mujeres y hombres, Ventura Franch, Asunción y García Campá, Santiago (dirs.), Navarra, Aranzadi.

CASAS BAAMONDE, MARÍA EMILIA (2019) "La igualdad de género en el Estado Constitucional”, Revista de Derecho Social, n. 88, pp. 13-54.

DOMÍNGUEZ MORALES, ANA (2018), Igualdad, no discriminación y negociación colectiva, Madrid, Ediciones Cinca.

ESQUEMBRE CERDÁ, MAR (2018) "El reconocimiento de las mujeres como sujetos jurídico-políticos en la Ley de Igualdad", pp. 91-117, El derecho de igualdad efectiva de mujeres y hombres, Ventura Franch, Asunción y García Campá, Santiago (dirs.), Navarra, Aranzadi.

FABREGAT MONFORT, GEMMA (2009) Las medidas de acción positiva. La posibilidad de una nueva tutela antidiscriminatoria, Valencia, Tirant Lo Blanch

FABREGAT MONFORT, GEMMA (2018-1) "planes y distintivos de igualdad en la empresa", pp. 743-767, El derecho de igualdad efectiva de mujeres y hombres, Ventura Franch, Asunción y García Campá, Santiago (dirs.), Navarra, Aranzadi.

FABREGAT MONFORT, GEMMA (2018-2) “La incidencia de la Ley orgánica 3/2007, de 22 de marzo, para la igualdad efectiva de mujeres y hombres en materia de negociación colectiva. En especial, en los planes de igualdad", pp. 369-390, Trabajo, Género e Igualdad, Romero Burillo, Ana María (dir. y coord.), Navarra, Aranzadi.

FABREGAT MONFORT, GEMMA (2019) La obligatoriedad del plan de igualdad tras el RDL6/2019, de 1 de marzo, Albacete, Bomarzo.

GARCÍA BLASCO, JUAN (dir.) (2019) La negociación colectiva en el sector público, Madrid, Ministerio de Trabajo, Migraciones y Seguridad Social.

GARCÍA CAMPÁ, SANTIAGO y VENTURA FRANCH, ASUNCIÓN (2018) "Hacia una nueva dogmática del derecho a la igualdad efectiva de mujeres y hombres", pp.51-90, El derecho de igualdad efectiva de mujeres y hombres, Ventura Franch, Asunción y García Campá, Santiago (dirs.), Navarra, Aranzadi.

GRAU PINEDA, CARMEN (2014) "Los planes de igualdad como ¿nueva? técnica para la consecución de la igualdad en la empresa", pp. 263-286, Estado de Derecho y discriminación por razón de género, orientación e identidad sexual. Cuesta López, Víctor Manuel y Santana Vega, Dulce María (coords.), Navarra, Aranzadi.

GRAU PINEDA, CARMEN (2020) "Los enemigos que amenazan la efectividad real de los planes de igualdad”, versión pendiente de publicación, Femeris, 2020. 
LOUSADA AROCHENA, JOSÉ F. (2011) “La dimensión objetiva del derecho a la igualdad de mujeres y hombres”, Aequalitas, n. 29, pp. 24-29.

LOUSADA AROCHENA, JOSÉ F. (2008) Permiso de paternidad y conciliación masculina, Albacete, Bomarzo.

LUJÁN ALCARAZ, JOSÉ y SELMA PENALVA, ALEJANDRA (2016) “planes de Igualdad”, pp. 455-492, El principio de igualdad en la negociación colectiva, Sánchez Trigueros, Carmen (Dir.), Madrid, Ministerio de Empleo y Seguridad Social.

MERINO SEGOVIA, AMPARO (2009) Igualdad de género, empresa y responsabilidad social, Albacete, Bomarzo.

MONEREO PÉREZ, JOSÉ LUIS y GUINDO MORALES, SARA (2018) “planes de Igualdad”, pp. 565-579, Un decenio de jurisprudencia laboral sobre la Ley de igualdad entre mujeres y hombres, Sánchez Trigueros, Carmen (dir.), Madrid, BOE.

OLMO GASCÓN, ANA MARTA (2013) "El contenido de los planes de igualdad: guías y directrices prácticas”, Aequalitas, n. 33, pp. 38-58.

POQUET CATALÁ, RAQUEL (2019) “Doctrina judicial en torno a la elaboración y negociación del plan de igualdad", Trabajo y Derecho, n. 54, pp. 89-97.

ROMERO RODENAS, MARÍA JOSÉ (2017), planes de igualdad, Albacete, Bomarzo.

SALDAÑA VALDERAS, EVA (2011) "El tratamiento del salario y los beneficios sociales en los planes de Igualdad: insuficiencias y buenas prácticas”, Temas Laborales, n. 108, pp. 85-113.

SALDAÑA VALDERAS, EVA (2013) “Los sistemas de clasificación profesional en los planes de igualdad de la empresa privada y del sector público", Temas Laborales, n. 119, pp. 91-118.

SÁNCHEZ TRIGUEROS, CARMEN (Dir.) (2016) El principio de igualdad en la negociación colectiva, Madrid, Ministerio de Empleo y Seguridad Social.

SELMA PENALVA, ALEJANDRA (2017) "Algunas reflexiones prácticas en torno a la elaboración de los planes de igualdad en las empresas", Revista General de Derecho del Trabajo y de la Seguridad Social, n. 46, versión digital.

SELMA PENALVA, ALEJANDRA (Dir.) (2019) Retos en materia de igualdad de género en el Siglo XXI, Madrid, Dykinson. 